\title{
Beam-Based Analog Self-Interference Cancellation with Auxiliary Transmit Chains in Full-Duplex MIMO Systems
}

\author{
Anh Tuyen Le ${ }^{1}$, Le Chung $\operatorname{Tran}^{2}$, Xiaojing Huang ${ }^{1}$, and Y. Jay Guo ${ }^{1}$ \\ ${ }^{1}$ University of Technology Sydney, Ultimo, NSW, 2007, Australia \\ Email: \{anhtuyen.le@student.uts.edu.au \\ Email: $\{x i a o j i n g \cdot h u a n g, j a y \cdot g u o\} @ u t s . e d u \cdot a u$ \\ ${ }^{2}$ University of Wollongong, Wollongong, NSW, 2522, Australia \\ Email: lctran@uow.edu.au
}

\begin{abstract}
Analog domain cancellation has been considered as the most important step to mitigate self-interference (SI) in fullduplex (FD) radios. However, in FD multiple-input multipleoutput (MIMO) systems, this method faces a critical issue of complexity since the number of cancellation circuits increases quadratically with the number of antennas. In this paper, we propose a beam-based radio frequency SI cancellation architecture which uses adaptive filters to significantly reduce the complexity. Data symbols for all the beams are up-converted by auxiliary transmit chains to provide reference signals for all adaptive filters. Hence, the number of cancellation circuits becomes proportional to the number of transmit beams which are much smaller than that of transmit antennas. We then show that the interference suppression ratio in this architecture is neither affected by the number of beams nor transmit or receive antennas. Instead, it is decided by the performance of the adaptive filter. Simulations are conducted to confirm the theoretical analyses.
\end{abstract}

Index Terms-Full-duplex, self-interference cancellation, ALMS loop, beamforming, and MIMO.

\section{INTRODUCTION}

Future generations of mobile communication networks are expected with many great advantages such as much faster data rate, much lower round trip latency, less power consumption and so on [1]. To enable these great features, multiple-input multiple-output (MIMO) beamforming and full-duplex (FD) are considered as key technologies [2]. In FD operations, transceivers can simultaneously transmit and receive signals on the same frequency band so that the throughput is doubled compared to half-duplex counterparts [3]. However, these great advantages come together with a critical issue of selfinterference (SI) which is the cross-talk between the co-located transmitter and receiver.

Self-interference can be mitigated through three stages in propagation domain, analog domain and digital domain [4]. Among them, analog domain cancellation has been proved as the most effective step because propagation approaches are limited by the size of devices, while digital domain cancellation is limited by the effective dynamic range of the analogto-digital converter [4]. For analog domain cancellation, the transmitted signal after the power amplifier is employed as reference signal whose phase and amplitude are modified to generate a cancellation signal which will subtract the SI at the input of the receiver. Some promising analog cancellation schemes can be found in [5]-[7].

However, it is very challenging to implement analog domain cancellation in FD MIMO beamforming systems due to complexity. If a cancellation circuit is required for each pair of transmit and receive antennas, an $N \times N$ FD MIMO system will need $N^{2}$ cancellation circuits [8]. Therefore, digital beamforming has been used in [9]-[11] to exploit spatial suppression. As pointed out in [12], this method reduces the transmission rate because some of the available spatial Degrees of Freedom are allocated for SI mitigation. Recent works have proposed analog domain cancellation for FD MIMO by a multi-tap architecture [12]-[14] or by precoding in digital baseband and additional transmit chains [15]. Since the channel state information is always required in these approaches, the FD MIMO has to operate in half-duplex modes sometimes to estimate the SI channel. In addition, since the complexity in the optimization problem depends on the cross-talk between a pair of transmit and receive antennas, the complexity of the digital signal processing algorithms increases quadratically with the size of MIMO systems [8].

In this paper, we propose a beam-based SI cancellation structure which employs analog least mean-square loops (ALMS) presented in [7] as adaptive filters. Since the ALMS loop is purely implemented in the radio frequency (RF) domain, no complicated digital signal processing is required. However, when applied in FD MIMO systems, the reference signals for all ALMS loops are generated in a different way compared to [7]. Instead of obtaining from the RF transmitted signal after the power amplifier in the transmit chain, the reference signals in this structure is generated by up-converting the transmitted data for all beams with auxiliary transmit chains. In this way, the number of cancellation circuits is reduced significantly because the number of beams is usually much smaller than the number of antennas. This reduction is 


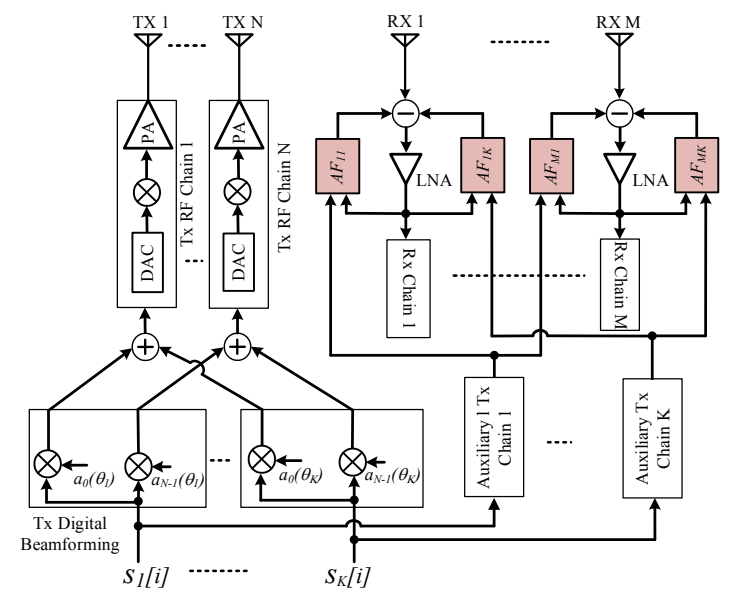

Fig. 1. A beam-based analog SI cancellation structure.

extremely helpful in massive FD MIMO. Stationary analysis is then applied to derive the SI and residual SI powers as well as the interference suppression ratio (ISR). By averaging over many realizations of SI channels, we show that the ISR is not affected by the number of transmit or receive antennas, but it is determined by the performance of the AMS loop which has been comprehensively investigated in [7], [16], [17].

The rest of this paper is organized as follows. In Section II, the beam-based cancellation architecture for FD MIMO and the signal models are described. In Section III, SI and residual SI powers as well as ISR are derived and examined through stationary analysis. In Section IV, simulations are conducted to verify the theoretical analysis. Finally, conclusions are drawn in Section V.

\section{SYSTEM DESCRIPTIONS}

\section{A. Beam-Based SI Cancellation}

Consider an FD MIMO digital beamforming system with $N$ transmit and $M$ receive antennas in Fig. 1. The transmit data symbol streams for $K$ directions, denoted as $s_{k}(i), i=$ $-\infty, \cdots, \infty, k=1, \cdots, K$, are multiplied with the corresponding array vector $\mathbf{a}\left(\theta_{k}\right)$ before added together and transmitted by $N$ transmit chains. The vector of RF transmitted signals at $N$ antennas at the time $t$ is expressed as

$$
\mathbf{x}(t)=\operatorname{Re}\left\{\left[X_{1}(t) \cdots X_{N}(t)\right]^{T} e^{j 2 \pi f_{c} t}\right\}
$$

where $X_{n}(t), n=1, \cdots, N$ are the baseband equivalents of these signals. The vector $\mathbf{X}(t)=\left[\begin{array}{llll}X_{1}(t) & \cdots & X_{N}(t)\end{array}\right]^{T}$ is expressed as

$$
\mathbf{X}(t)=\mathbf{A} \mathbf{S}(t)=\left[\begin{array}{ccc}
a_{0}\left(\theta_{1}\right) & \cdots & a_{0}\left(\theta_{K}\right) \\
\vdots & \ddots & \vdots \\
a_{N-1}\left(\theta_{1}\right) & \cdots & a_{N-1}\left(\theta_{K}\right)
\end{array}\right]\left[\begin{array}{c}
S_{1}(t) \\
\vdots \\
S_{K}(t)
\end{array}\right]
$$

where $a_{n}\left(\theta_{k}\right)=e^{j n \frac{2 \pi}{\lambda} d \sin \theta_{k}}, n=0, \cdots, N-1$ is the array vector of the beam at direction $\theta_{k}, d$ is the distance between

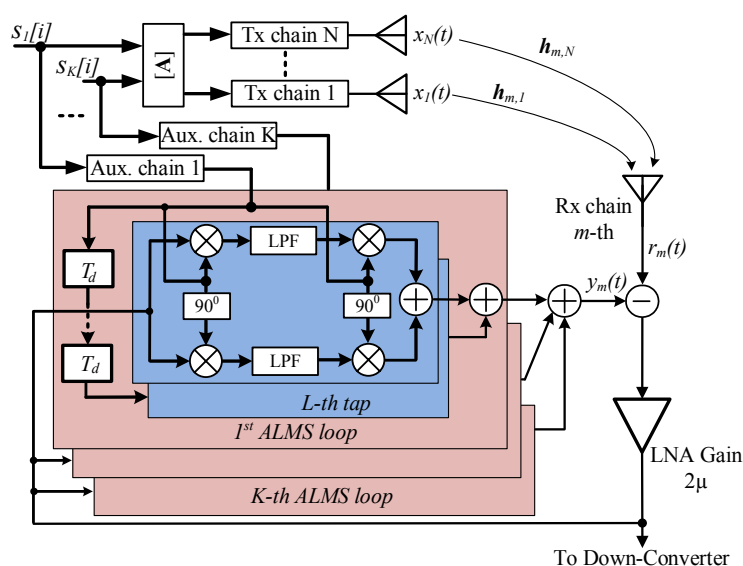

Fig. 2. $K$ ALMS loops at the $m$-th receiver chain.

antenna elements; $S_{k}(t)=\sum_{i=-\infty}^{\infty} s_{k}(i) p\left(t-i T_{s}\right)$ is the lowpass equivalent of the transmitted signal at the $k$-th direction and $p(t)$ is the pulse shaping filter. Assuming that the data for each direction are independent, .i.e.,

$$
E\left\{s_{k}(i)^{*} s_{k^{\prime}}\left(i^{\prime}\right)\right\}=\left\{\begin{array}{l}
1, \text { for } k=k^{\prime} \text { and } i=i^{\prime} \\
0, \text { for } k \neq k^{\prime} \text { or } i \neq i^{\prime}
\end{array}\right.
$$

where $E\{$.$\} stands for ensemble expectation.$

Due to the FD operation, at the $m$-th receiver chain, there will present of the SI from the co-located transmitters $z_{m}(t)$, the desired signal $s_{m}^{r}(t)$, and noise $n_{m}(t)$ with $m=1, \cdots, M$. To mitigate the SI, $K$ adaptive filters are employed at each receiver chain. The reference signals for these adaptive filters are generated from $K$ data streams by corresponding additional transmit chains which are assumed to be the same as those in the main transmission paths. The outputs from $K$ cancellation circuits are added together to cancel the SI at the input of the considered receiver chain.

\section{B. ALMS Loop}

Each adaptive filter is implemented by an ALMS loop which uses a multi-tap mechanism as illustrated in Fig. 2. Considering the $k$-th ALMS loop at $m$-th receiver chain, the reference signal generated by the auxiliary chain of the beam $k$-th is passed into the ALMS loop which includes $L$ taps. At the $l$-th tap, the reference signal is delayed by $(l-1) T_{d}$, where $T_{d}$ is the tap delay, before multiplied by the amplified residual signal $d_{m}(t)$. For simplicity, the losses caused by power splitters for both reference and loop-back signals are normalized to one. The outputs of the multiplier are filtered by a ResistorCapacitor low-pass filter to generate a weighting coefficient $w_{m, k}(l, t)$ which will modify another version of the delayed reference signal. The signals at output of all taps are added together to obtain the cancellation signal $y_{m}(t)$ to subtract the SI $z_{m}(t)$. The residual signal is amplified and looped-back to the input of all adaptive filters. Assuming that the SI channel between a pair of transmit and receive antennas includes $L$ taps with the tap delay $T_{d}$ equal to that in the ALMS loop. 
The vector of SI signals $\mathbf{z}(t)=\left[z_{1}(t), \cdots, z_{M}(t)\right]^{T}$ appearing at $M$ receiver chains is presented by

$$
\mathbf{z}(t)=\operatorname{Re}\left\{\sum_{l=0}^{L-1} \mathbf{H}^{*}(l) \mathbf{X}\left(t-l T_{d}\right) e^{j 2 \pi f_{c} t}\right\}
$$

where $\mathbf{H}(l)=\left[h_{m, n}(l)\right], m=1, \cdots, M, n=1, \cdots, N$, is the l-tap of $M \times N$ SI channel coefficients matrix and $\mathbf{H}^{*}$ denote the complex conjugate of all elements of the matrix $\mathbf{H}$.

At the input of the $M$ receiver chains, the vector of received signals is expressed as $\mathbf{r}(t)=$ $\left[\operatorname{Re}\left\{\left[Z_{1}(t)+S_{1}^{r}(t)+N_{1}(t)\right] e^{j 2 \pi f_{c} t}\right\}, \cdots, \operatorname{Re}\left\{\left[Z_{M}(t)+\right.\right.\right.$ $\left.\left.\left.S_{M}^{r}(t)+N_{M}(t)\right] e^{j 2 \pi f_{c} t}\right\}\right]^{T}$ where $Z_{m}(t), S_{m}^{r}(t)$, and $N_{m}(t)$ are the low-pass equivalents of the SI signal, desired signal and additive Gaussian noise at the $m$-th receiver chain. To cancel the SI, a vector of cancellation signals at all $M$ receiver chains are generated as

$$
\mathbf{y}(t)=\operatorname{Re}\left\{\sum_{l=0}^{L-1} \mathbf{W}^{*}(l, t) \mathbf{S}\left(t-l T_{d}\right) e^{j 2 \pi f_{c}\left(t-l T_{d}\right)}\right\}
$$

where $\mathbf{W}(l, t)=\left[w_{m, k}(l, t)\right], m=1, \cdots, M, k=1, \cdots, K$ is the $M \times K$ weighting coefficient matrix, and $w_{m, k}(l, t)$ is the weighting coefficient of the $l$-th tap in the $k$-th ALMS loop at the $m$-th receiver chain. Similar to Eq. (5) in [7], $\mathbf{W}(l, t)$ is calculated by

$$
\begin{gathered}
\mathbf{W}\left(l, t+t_{0}\right)=\frac{2 \mu \alpha}{K_{1} K_{2}} \int_{t_{0}}^{t+t_{0}} e^{-\alpha(t-\tau)}[\mathbf{r}(\tau)-\mathbf{y}(\tau)] \\
\cdot \mathbf{S}^{T}\left(\tau-l T_{d}\right) e^{j 2 \pi f_{c}\left(\tau-l T_{d}\right)} d \tau
\end{gathered}
$$

where $K_{1}$ and $K_{2}$ are the dimensional constants of the multipliers, $2 \mu$ is the gain of the LNA, $\alpha=1 / R C$ is the decay constant of the LPF with resistance $R$ and capacitance $C$, and $0 \leq t_{0}<T_{s}$ is an initial starting time.

\section{StationARY ANALYsis}

In this section, we apply stationary analysis to analyze the convergence behaviors of all ALMS loops. For stationary analysis, both ensemble expectation and time averaging operations, denoted as $\bar{E}\{$.$\} , are applied to evaluate the random processes.$ The normalized auto-correlation functions of the transmitted signals are defined as

$$
\begin{aligned}
\Phi_{k, k^{\prime}}(\tau) & =\bar{E}\left\{S_{k}^{*}(t) S_{k^{\prime}}(t-\tau)\right\} \\
& =\frac{1}{K_{1} K_{2} T_{s}} \int_{0}^{T_{s}} E\left\{S_{k}^{*}(t) S_{k^{\prime}}(t-\tau)\right\} d t .
\end{aligned}
$$

Applying the assumption in (3) into (7), we have

$$
\Phi_{k, k^{\prime}}(\tau)= \begin{cases}\Phi_{k}(\tau) & \text { for } k=k^{\prime} \\ 0 & \text { for } k \neq k^{\prime}\end{cases}
$$

where $\Phi_{k}(\tau)=\frac{1}{K_{1} K_{2} T_{s}} \int_{0}^{T_{s}} p^{*}(t) p(t-\tau) d t$ is the normalized autocorrelation function of the transmitted signal at the $k$-th beam. Since all the transmit chains in both main paths and auxiliary paths are assumed to be the same, we have $\Phi_{k}(\tau)=$ $\Phi_{k^{\prime}}(\tau)=\Phi(\tau)$.

The SI power at the $m$-th receiver chain is calculated as

$$
\begin{aligned}
& P_{I_{m}}(t)=\frac{1}{K_{1} K_{2}} \bar{E}\left\{z_{m}^{2}(t)\right\}=\frac{1}{K_{1} K_{2}} \times \\
& \bar{E}\left\{\left[\operatorname{Re}\left\{\sum_{l=0}^{L-1} \mathbf{h}_{m}^{*}(l) \mathbf{X}\left(t-l T_{d}\right) e^{j 2 \pi f_{c} t}\right\}\right]^{2}\right\}=\frac{1}{2 K_{1} K_{2}} \times \\
& \bar{E}\left\{\sum_{l=0}^{L-1} \sum_{l^{\prime}=0}^{L-1}\left[\mathbf{h}_{m}^{*}(l) \mathbf{X}\left(t-l T_{d}\right)\right]\left[\mathbf{h}_{m}^{*}\left(l^{\prime}\right) \mathbf{X}\left(t-l^{\prime} T_{d}\right)\right]^{H}\right\} \\
& =\frac{1}{2 K_{1} K_{2}} \sum_{l=0}^{L-1} \sum_{l^{\prime}=0}^{L-1} \mathbf{h}_{m}^{*}(l) \mathbf{A} \bar{E}\left\{\mathbf{S}\left(t-l T_{d}\right) \mathbf{S}^{H}\left(t-l^{\prime} T_{d}\right)\right\} \\
& \cdot \mathbf{A}^{H} \mathbf{h}_{m}^{T}\left(l^{\prime}\right)=\frac{1}{2} \sum_{l=0}^{L-1} \sum_{l^{\prime}=0}^{L-1} \mathbf{h}_{m}^{*}(l) \mathbf{A} \boldsymbol{\Theta}\left(\left(l-l^{\prime}\right) T_{d}\right) \mathbf{A}^{H} \mathbf{h}_{m}^{T}\left(l^{\prime}\right)
\end{aligned}
$$

where $\mathbf{h}_{m}(l)=\left[h_{m, 1}(l), \cdots, h_{m, N}(l)\right], \boldsymbol{\Theta}\left(\left(l-l^{\prime}\right) T_{d}\right)=$ $\Phi\left(\left(l-l^{\prime}\right) T_{d}\right) \mathbf{I}_{K}$ where $\mathbf{I}_{K}$ is the identity matrix of size $K$. Extending (9) in the full form of $L$ and $M$, we have the total SI power at all $M$ receiver chains is expressed as

$$
P_{I}(t)=\sum_{m=1}^{M} P_{I_{m}}(t)=\frac{1}{2} \underline{\mathbf{h}}^{H}\left[\mathbf{I}_{M} \otimes \boldsymbol{\Theta}\right] \underline{\mathbf{h}}
$$

where $\underline{\mathbf{h}}=\left[\mathbf{I}_{M} \otimes\left(\mathbf{A}^{H} \otimes \mathbf{I}_{L}\right)\right] \mathbf{h}, \mathbf{h}=\left[h_{1,1}(0), \cdots, h_{1,1}(L-\right.$ $\left.1), \cdots, h_{M, N}(L-1)\right]^{T}$, and $\boldsymbol{\Theta}=\boldsymbol{\Phi} \otimes \mathbf{I}_{K}, \boldsymbol{\Phi}=$

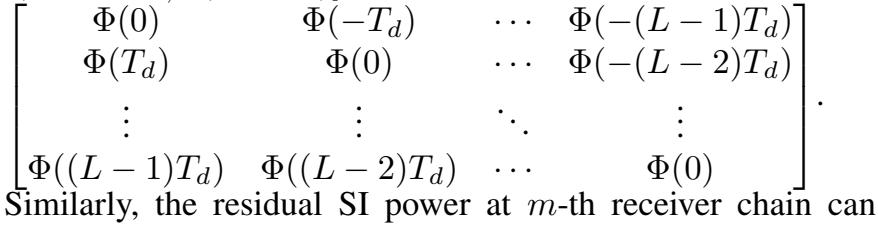
be calculated as

$$
\begin{aligned}
& P_{R I_{m}}(t)=\frac{1}{K_{1} K_{2}} \bar{E}\left\{\left[z_{m}(t)-y_{m}(t)\right]^{2}\right\}=\frac{1}{2 K_{1} K_{2}} \times \\
& \bar{E}\left\{\left[\sum_{l=0}^{L-1}\left[\mathbf{h}_{m}^{*}(l) \mathbf{A}-\mathbf{w}_{m}^{*}(l, t) e^{-j 2 \pi f_{c} l T_{d}}\right] \mathbf{S}\left(t-l T_{d}\right)\right]^{2}\right\} .
\end{aligned}
$$

Denote $\mathbf{u}_{m}(l, t)=\left[u_{m, 1}(l, t), \cdots u_{m, K}(l, t)\right]=\mathbf{h}_{m}(l) \mathbf{A}^{*}-$ $\mathbf{w}_{m}(l, t) e^{j 2 \pi f_{c} l T_{d}}$ as the vector of weighting error functions which shows the difference between the weighting coefficients of the $l$-th tap in $K$ ALMS loops and the channel coefficients of the same tap in the SI channel at the $m$-th receiver chain. The expression of $P_{R I_{m}}(t)$ becomes

$$
\begin{aligned}
& P_{R I_{m}}(t)=\frac{1}{2 K_{1} K_{2}} \bar{E}\left\{\left[\sum_{l=0}^{L-1} \mathbf{u}_{m}^{*}(l, t) \mathbf{S}\left(t-l T_{d}\right)\right]^{2}\right\} \\
& =\frac{1}{2 K_{1} K_{2}} \bar{E}\left\{\sum_{l=0}^{L-1} \mathbf{u}_{m}^{*}(l, t) \mathbf{S}\left(t-l T_{d}\right) \sum_{l^{\prime}=0}^{L-1} \mathbf{S}^{H}\left(t-l^{\prime} T_{d}\right)\right. \\
& \left.\cdot \mathbf{u}_{m}^{T}\left(l^{\prime}, t\right)\right\}=\frac{1}{2} \sum_{l=0}^{L-1} \sum_{\substack{l^{\prime}=0 \\
l^{\prime} \neq l}}^{L-1} \overline{\mathbf{u}}_{m}^{*}(l, t) \Theta\left(\left(l-l^{\prime}\right) T_{d}\right) \overline{\overline{\mathbf{u}}}_{m}^{T}\left(l^{\prime}, t\right) \\
& +\frac{1}{2} \Phi(0) \sum_{l=0}^{L-1} \sum_{k=1}^{K} \overline{\bar{u}}_{m, k}^{2}(l, t)
\end{aligned}
$$


where $\quad \overline{\mathbf{u}}_{m}(l, t)=\bar{E}\left\{\mathbf{u}_{m}(l, t)\right\} \quad$ and $\quad \overline{\bar{u}}_{m, k}^{2}(l, t)=$ $\bar{E}\left\{u_{m, k}^{2}(l, t)\right\}$. Hence, the total residual SI power of all $M$ receiver chains is represented by

$$
\begin{aligned}
P_{R I}(t)= & \sum_{m=1}^{M} P_{R I_{m}}(t) \\
= & \frac{1}{2} \overline{\overline{\mathbf{u}}}^{H}(t)\left[\mathbf{I}_{M} \otimes \boldsymbol{\Theta}-\Phi(0) \mathbf{I}_{M K L}\right] \overline{\overline{\mathbf{u}}}(t) \\
& +\frac{1}{2} \Phi(0) \sum_{m, k, l} \overline{\bar{u}}_{m, k}^{2}(l, t)
\end{aligned}
$$

where $\overline{\overline{\mathbf{u}}}(t)=\left[\overline{\bar{u}}_{1,1}(0, t), \cdots \overline{\bar{u}}_{1,1}(L-1, t), \cdots, \overline{\bar{u}}_{M, K}(L-\right.$ $1, t)]^{T}$. From (13), we need to derive the vector $\overline{\overline{\mathbf{u}}}(t)$ to evaluate the performance of the adaptive filters.

Representing the definition of the weighting error functions in the matrix form, i.e., $\mathbf{U}(l, t)=\mathbf{H}_{l} \mathbf{A}^{*}-\mathbf{W}(l, t) e^{j 2 \pi f_{c} l T_{d}}$ where $\mathbf{U}(l, t)$ is a $M \times K$ matrix of $u_{m, k}(l, t)$. From (6), we have

$$
\begin{aligned}
& \mathbf{U}\left(l, t+t_{0}\right)=\mathbf{H}_{l} \mathbf{A}^{*}-\frac{\mu \alpha}{K_{1} K_{2}} \int_{t_{0}}^{t+t_{0}} e^{-\alpha(t-\tau)} \times \\
& {\left[\sum_{l^{\prime}=0}^{L-1} \mathbf{U}\left(l^{\prime}, \tau\right) \mathbf{S}^{*}\left(\tau-l^{\prime} T_{d}\right)+\mathbf{S}_{r}^{*}(\tau)+\mathbf{N}^{*}(\tau)\right] \mathbf{S}^{T}\left(\tau-l T_{d}\right) d \tau .}
\end{aligned}
$$

Assuming that the transmitted signals are independent to the desired signals and noise, applying both ensemble expectation and time average over one $T_{s}$ with respect to the starting time $t_{0}$ for both sides of (14), we obtain

$$
\overline{\overline{\mathbf{U}}}(l, t)=\mathbf{H}_{l} \mathbf{A}^{*}-\mu \alpha \int_{0}^{t} e^{-\alpha(t-\tau)} \sum_{l^{\prime}=0}^{L-1} \overline{\overline{\mathbf{U}}}\left(l^{\prime}, \tau\right) \Theta\left(\left(l-l^{\prime}\right) T_{d}\right) d \tau
$$

Applying vectorization to both sides of (15) and then extending to the full form of L-taps, (15) becomes

$$
\overline{\overline{\mathbf{u}}}(t)=\underline{\mathbf{h}}-\mu \alpha \int_{0}^{t} e^{-\alpha(t-\tau)}\left[\mathbf{I}_{M} \otimes \boldsymbol{\Theta}^{T}\right] \overline{\overline{\mathbf{u}}}(\tau) d \tau .
$$

Denote $\boldsymbol{\Psi}=\mathbf{I}_{M} \otimes \boldsymbol{\Theta}^{T}$ and note that $\boldsymbol{\Psi}$ can be decomposed as $\boldsymbol{\Psi}=\mathrm{Q} \Lambda \mathbf{Q}^{-1}$ where $\mathrm{Q}$ is the orthonormal modal matrix whose columns are the $M \times K \times L$ eigenvectors of $\Psi$, and $\Lambda$ is a diagonal matrix whose diagonal includes eigenvalues $\lambda_{i}, i=1, \cdots, M \times K \times L$ of $\boldsymbol{\Psi}$. Following the steps shown in [7], we obtain the solution of the weighting error functions as

$$
\begin{aligned}
& \overline{\overline{\mathbf{u}}}(t)=\mathbf{Q} \operatorname{diag}\left\{\frac{1}{1+\mu \lambda_{i}}+\frac{\mu \lambda_{i}}{1+\mu \lambda_{i}} e^{-\left(1+\mu \lambda_{i}\right) \alpha t}\right\} \mathbf{Q}^{-1} \underline{\mathbf{h}} \\
& \sum_{m, k, l} \overline{\bar{u}}_{m, k}^{2}(l, t)=\underline{\mathbf{h}}^{H} \mathbf{Q} \operatorname{diag}\left\{\frac{1}{\left(1+\mu \lambda_{i}\right)^{2}}+\frac{2 \mu \lambda_{i}}{\left(1+\mu \lambda_{i}\right)^{2}} \times\right. \\
& \left.e^{-\left(1+\mu \lambda_{i}\right) \alpha t}+\frac{\left(\mu \lambda_{i}\right)^{2} e^{-2\left(1+\mu \lambda_{i}\right) \alpha t}}{\left(1+\mu \lambda_{i}\right)^{2}}-e^{-2(1+\mu \bar{\lambda}) \alpha t}\right\} \mathbf{Q}^{-1} \underline{\mathbf{h}}
\end{aligned}
$$

where $\bar{\lambda}=\Phi(0)$.

To evaluate the performance of the proposed structure under different scenarios, we define the interference suppression ratio (ISR) as $I S R(t)=P_{R I}(t) / P_{I}(t)$. From (10), (13), and (17),

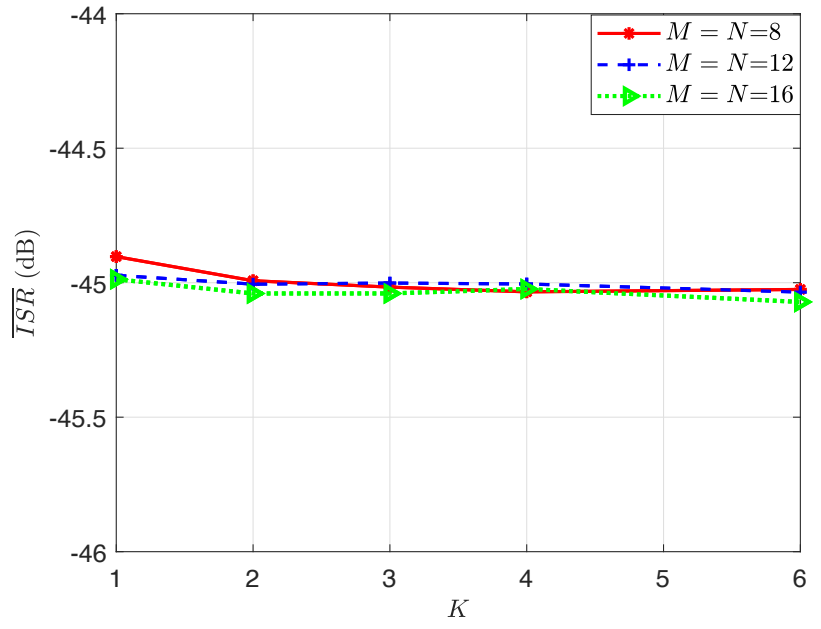

Fig. 3. The averaged and converged interference suppression ratio.

when $t \rightarrow \infty, I S R(t)$ converges to its stable value, denoted as $I S R$

$$
I S R=\frac{\underline{\mathbf{h}}^{H} \mathbf{Q} \operatorname{diag}\left\{\frac{\lambda_{i}}{\left(1+\mu \lambda_{i}\right)^{2}}\right\} \mathbf{Q}^{-1} \underline{\mathbf{h}}}{\underline{\mathbf{h}}^{H} \mathbf{Q} \operatorname{diag}\left\{\lambda_{i}\right\} \mathbf{Q}^{-1} \underline{\mathbf{h}}} .
$$

To evaluate $I S R$ over many realizations of the SI channel, we define the averaged interference suppression ratio $\overline{I S R}=\bar{E}_{h}\{I S R\}$ where $\bar{E}_{h}$ stands for expectation over the SI channel. Fig. 3 presents $\overline{I S R}$ obtained by averaging over 1000 realizations of the SI channel whose coefficients are assumed to be independent and have Gaussian distribution with zero mean. We can see that $\overline{I S R}$ is almost stable with different number of transmit and receive antennas as well as the number of beams. It means that the beam-based cancellation architecture works as expected and the level of SI mitigation can be determined by the performance of the adaptive filters.

\section{Simulation Results}

The simulations are performed for beamforming FD MIMO systems with the distance between antenna elements is selected at a half of wave-length. The QPSK modulated data symbols for all beams have duration of $T_{s}=20 \mathrm{~ns}$ and the carrier frequency is $f_{c}=5 \mathrm{GHz}$. The transmit chains in both main paths and auxiliary paths employ root-raised cosine filters with roll-off factors $\beta=0.25$. The power of the transmitted signal per antenna is $0 \mathrm{dBm}$, and the multiplier dimensional constants are $K_{1} K_{2}=0.001 V^{2}$. The LNA gain $2 \mu$ in all the receiver chains is selected as $\mu=10$. The ALMS loop is designed with $L=8$ taps and the tap delay is selected as $T_{d}=T_{s} / 2$. The SI channel between a pair of transmit and receive antenna is modeled with $L=8$ taps and the propagation delay of each tap is the same as that in the ALMS loop. The propagation losses of all SI channels are set to be $25 \mathrm{~dB}$.

In the first simulation, an $8 \times 8$ FD MIMO system is simulated with two cases of the number of beams $K=2$ and $K=4$. In the second simulation, the number of beams is fixed at $K=2$ for a $4 \times 4$ and $8 \times 8$ FD MIMO systems. 


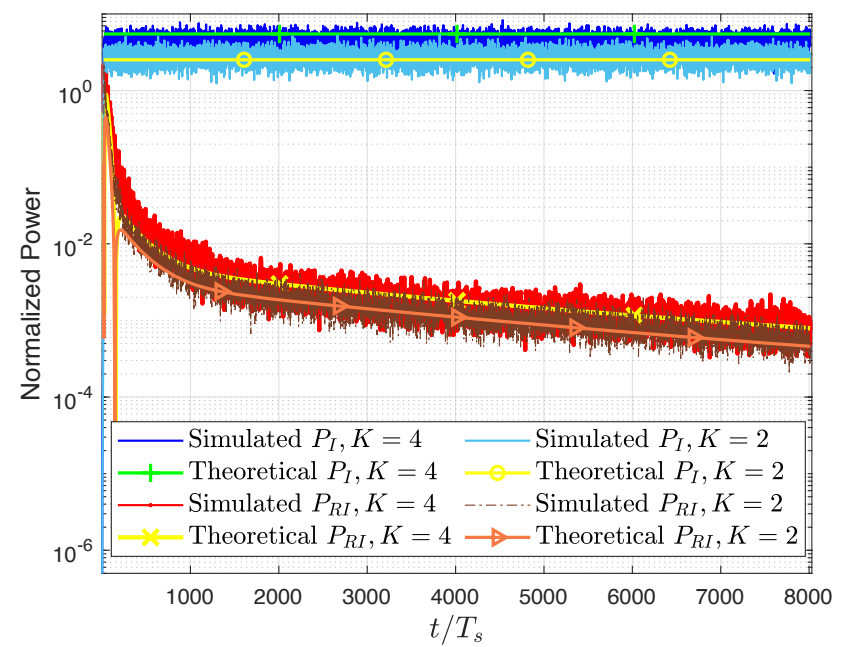

(a)

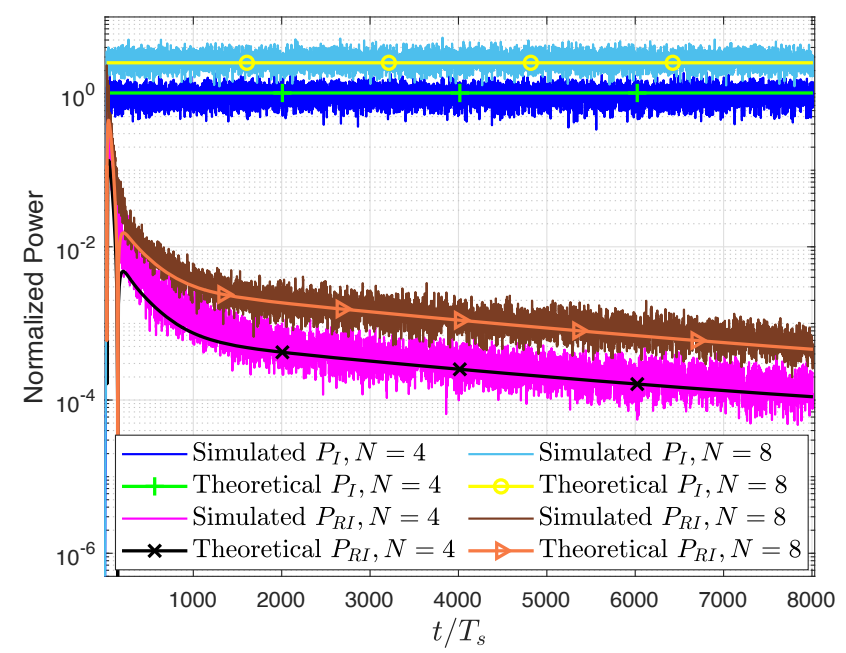

(b)

Fig. 4. Normalized SI and residual SI powers for (a) $8 \times 8$ FD MIMO systems and (b) 2 beams FD MIMO systems.

The SI powers and residual SI powers in the first and second simulations are plotted in Fig. 4 (a) and (b), respectively. From these figures, the theoretical results match the simulated results in all cases which confirm the theoretical analyses presented in Section III. We can also see that the level of the SI power increases with the number of beams and transmit antennas. However, the ISRs in all cases are almost the same at $t=8000 T_{s}$.

\section{CONCLUSION}

In this paper, we have presented a novel beam-based analog SI cancellation architecture for FD MIMO systems. By using up-converted beam data as reference signals for the ALMS loops, the number of cancellation circuits is significantly reduced to be proportional to the number of transmit beams. Theoretical analyses and simulation results show that the level of cancellation is determined by the performance of the ALMS loop rather than the quantities of beams as well as transmit and receive antennas.

\section{ACKNOWLEDGMENT}

This work was supported by the Australian Research Council (DP160101693).

\section{REFERENCES}

[1] J. G. Andrews et al., "What will 5G be?" IEEE Journal on Selected Areas in Communications, vol. 32, no. 6, pp. 1065-1082, June 2014.

[2] M. Agiwal, A. Roy, and N. Saxena, "Next generation 5G wireless networks: A comprehensive survey," vol. 18, no. 3, pp. 1617-1655, 2016

[3] S. Goyal et al., "Full duplex cellular systems: will doubling interference prevent doubling capacity?" IEEE Communications Magazine, vol. 53, no. 5, pp. 121-127, May 2015.

[4] A. Sabharwal et al., "In-band full-duplex wireless: Challenges and opportunities," IEEE J. Sel. Areas Commun, vol. 32, no. 9, pp. 16371652, Sept 2014.

[5] K. E. Kolodziej, J. G. McMichael, and B. T. Perry, "Multitap RF canceller for in-band full-duplex wireless communications," IEEE Trans. Wireless Commun., vol. 15, no. 6, pp. 4321-4334, June 2016.
[6] A. Kiayani et al., "Adaptive nonlinear RF cancellation for improved isolation in simultaneous transmit-receive systems," IEEE Trans. Microw. Theory Techn., vol. 66, no. 5, pp. 2299-2312, Jan. 2018.

[7] X. Huang and Y. J. Guo, "Radio frequency self-interference cancellation with analog least mean-square loop," IEEE Trans. Microw. Theory Tech. vol. 65, no. 9, pp. 3336-3350, Sept. 2017.

[8] D. Bharadia and S. Katti, "Full duplex MIMO radios," in Proceedings of the 11th USENIX Conference on Networked Systems Design and Implementation, ser. NSDI'14. Berkeley, CA, USA: USENIX Association, 2014, pp. 359-372. [Online]. Available: http://dl.acm.org/citation.cfm?id=2616448.2616482

[9] T. Snow, C. Fulton, and W. J. Chappell, "Transmitreceive duplexing using digital beamforming system to cancel self-interference," IEEE Transactions on Microwave Theory and Techniques, vol. 59, no. 12, pp. 3494-3503, Dec 2011.

[10] T. Riihonen, S. Werner, and R. Wichman, "Mitigation of loopback selfinterference in full-duplex MIMO relays," IEEE Transactions on Signal Processing, vol. 59, no. 12, pp. 5983-5993, Dec 2011.

[11] E. Everett, C. Shepard, L. Zhong, and A. Sabharwal, "Softnull: Manyantenna full-duplex wireless via digital beamforming," IEEE Transactions on Wireless Communications, vol. 15, no. 12, pp. 8077-8092, Dec 2016.

[12] G. C. Alexandropoulos and M. Duarte, "Joint design of multi-tap analog cancellation and digital beamforming for reduced complexity full duplex MIMO systems," in 2017 IEEE International Conference on Communications (ICC), May 2017, pp. 1-7.

[13] Y. Liu, D. Liu, X. Li, and C. Huang, "Multi-tap analog MIMO self-interference cancellation for full-duplex communications," in 2017 9th International Conference on Wireless Communications and Signal Processing (WCSP), Oct 2017, pp. 1-6.

[14] H. Iimori and G. Thadeu Freitas de Abreu, "Two-way full-duplex MIMO with hybrid TX-RX MSE minimization and interference cancellation," in 2018 IEEE 19th International Workshop on Signal Processing Advances in Wireless Communications (SPAWC), June 2018, pp. 1-5.

[15] S. Huberman and T. Le-Ngoc, "MIMO full-duplex precoding: A joint beamforming and self-interference cancellation structure," IEEE Transactions on Wireless Communications, vol. 14, no. 4, pp. 2205-2217, April 2015

[16] A. T. Le, L. C. Tran, and X. Huang, "Cyclostationary analysis of analog least mean square loop for self-interference cancellation in in-band fullduplex systems," IEEE Commun. Lett., vol. 21, no. 12, pp. 2738-2741, Sept 2017.

[17] A. T. Le et al., "Frequency domain characterization and performance bounds of ALMS loop for RF self-interference cancellation," IEEE Trans. Commun., vol. 67, no. 1, pp. 682-692, Jan. 2019. 